\title{
Exploring factors contributing to young workers' vulnerability to work-related harm
}

\author{
MANFRED MANGLICMOT*, OLIVIA COTTON**, SONIA CHEN*** and JOANNE \\ CRAWFORD ${ }^{* * * * *}$
}

\begin{abstract}
New Zealand has experienced significant increases in youth employment rates in the last 20 years with 40 per cent of people employed part-time. This age group has been associated with the second-highest rate of injury claims. At the current time, there is limited information on why young workers in New Zealand are more vulnerable to work-related harm. This project aimed to explore this with a convenience sample of young workers using an online survey. Participants represented a diverse ethnic population but the sample was predominately female. In total, 32.7 per cent of respondents had received no occupational health and safety (OHS) training when starting work. Fifty-seven per cent of the sample thought that OHS was valued by their employer. Interestingly, 63 per cent of the sample said they would be confident about speaking up about an OHS issue with most being willing to speak to their manager. In relation to stress, the analysis identified that there was an association between feeling stressed and being unable to follow OHS protocols $(\mathrm{p}=0.05)$. While this was a small exploratory study, suggestions made to improve OHS include clear and open communication between workers and employers, improved access to OHS resources and continuous and in-depth training.
\end{abstract}

Keywords: young workers, youth, work-related harm, occupational health and safety, stress, training

\section{Introduction}

New Zealand has experienced significant increases in youth employment rates in the last 20 years and currently has one of the lowest youth unemployment rates in the OECD (Department of Labour, 2007). According to Statistics New Zealand, 40 per cent of all employed young people are in part-time employment; one third of which are employed in retail trade, accommodation and food services. This equates to approximately 109,000 workers (Statistics New Zealand, 2020a).

\footnotetext{
* Victoria University of Wellington, New Zealand

${ }^{* *}$ Victoria University of Wellington, New Zealand

${ }^{* * *} \mathrm{ACC}$

***** Worksafe New Zealand Chair in Health and Safety, School of Health Victoria University of Wellington, New Zealand
}

\section{Acknowledgements}

The authors would like to thank ACC for sponsoring two Summer Scholarship students at Victoria University of Wellington

\section{Ethical Clearance}

Ethical clearance was applied for through the Victoria University of Wellington Human Ethics Committee. Application Number 29185

Author contact - Joanne Crawford joanne.crawford@vuw.ac.nz 
Young people are more likely to transition in and out of the labour workforce than other age groups. They are more likely to move around within the workforce and have a higher worker turnover rate. Between 2007 and 2011, one in four young workers changed their workplace in any quarter, compared with one in seven for people in other age groups (Statistics New Zealand, 2020a).

Research shows that the group aged between 15 and 24 years has the second-highest rate of workrelated injury claims with 133 claims per 1,000 full-time equivalent employees (Statistics New Zealand, 2020b). In 2019, young workers in the trade industry had 41,500 claims; the highest of any industry. This indicates that, for whatever reason, there are issues in maintaining young workers' health and safety.

International research has also identified this. Hanvold, et al. (2018), in their systematic review of young workers in the Nordic countries, identified that young unskilled workers were more vulnerable to accidental injury; and skilled workers and apprentices were more at risk of work-related illness. The authors highlight that young workers are not a homogenous group which makes managing their risks more complex.

Clarkson et al. (2017) surveyed young workers (aged 12-25 years) in South Australia. While these participants did identify OHS issues, including stress, fatigue and heavy lifting, they also lacked confidence in reporting OHS issues and had limited information on where to seek help.

Young people often work in industries that do not provide adequate health and safety training, leading to young workers being unprepared to engage in safe workplace practice (Bohle et al., 2008). Therefore, it is likely that younger workers do not receive adequate support and knowledge to ensure safe working practices, increasing their vulnerability to work-related harm (Auckland Co-Design Lab, 2016).

Health and safety also incorporates psychosocial risks and stress. Research shows that stress was identified as a major risk to young workers' mental and emotional health and safety (Clarkson et al., 2018). Additionally, a quarter of young workers had experienced bullying at work (Clarkson et al., 2018). However, a majority were not confident enough to report health and safety issues to the appropriate people (e.g. managers, health and safety representatives, industry associations). This indicates key issues that negatively impact their ability to feel healthy and safe at work.

Currently, there lacks research providing explanations, from a young person's perspective, on why they feel that they may be more vulnerable to work-related harm. It is well-documented that they are more likely to be injured, but much of the research focuses on employers rather than seeking to understand the voice of young workers. There is also very limited research on the topic of young workers in New Zealand from the perspective of the young worker.

This exploratory study aimed to examine the experience of young workers in New Zealand in order to help understand what factors contribute to young workers' vulnerability to workplace-related harm.

\section{Method}

A 34-question online survey was developed based on the current MBIE (2018) research strategy. The survey was piloted using Google forms as an entry point. After ethical approval, the survey went live for 10 days. Recruitment to the survey was targeted, non-random sampling, advertising the survey on Facebook, Instagram, Safeguard magazine, Twitter, LinkedIn and using advertisements at Victoria 
University of Wellington. The survey was completed anonymously and was divided into six sections as below:

- Demographic information

- Health and safety training

- Prioritisation of worker health and safety

- Confidence of the participant

- Safety protocols and measurement

- Stress

The final question was an open-ended text box where participants could provide ideas that would make them feel safer at work. An entry into a prize draw for one of two $\$ 50$ supermarket vouchers was offered as incentive to take part. The data were collated and analysed using Excel and SPSS.

\section{Results}

\section{Demographics}

Overall, 108 people participated in the survey with a total of 107 usable responses. Table 1 shows the breakdown of the participants in relation to age, gender and ethnicity. As can be seen from the table, over 70 per cent of participants were female. Eighty-six per cent of participants were working at the time of the survey and the remaining 14 per cent had worked in the previous six months.

Table 1 Demographic breakdown of the sample

16-18 years

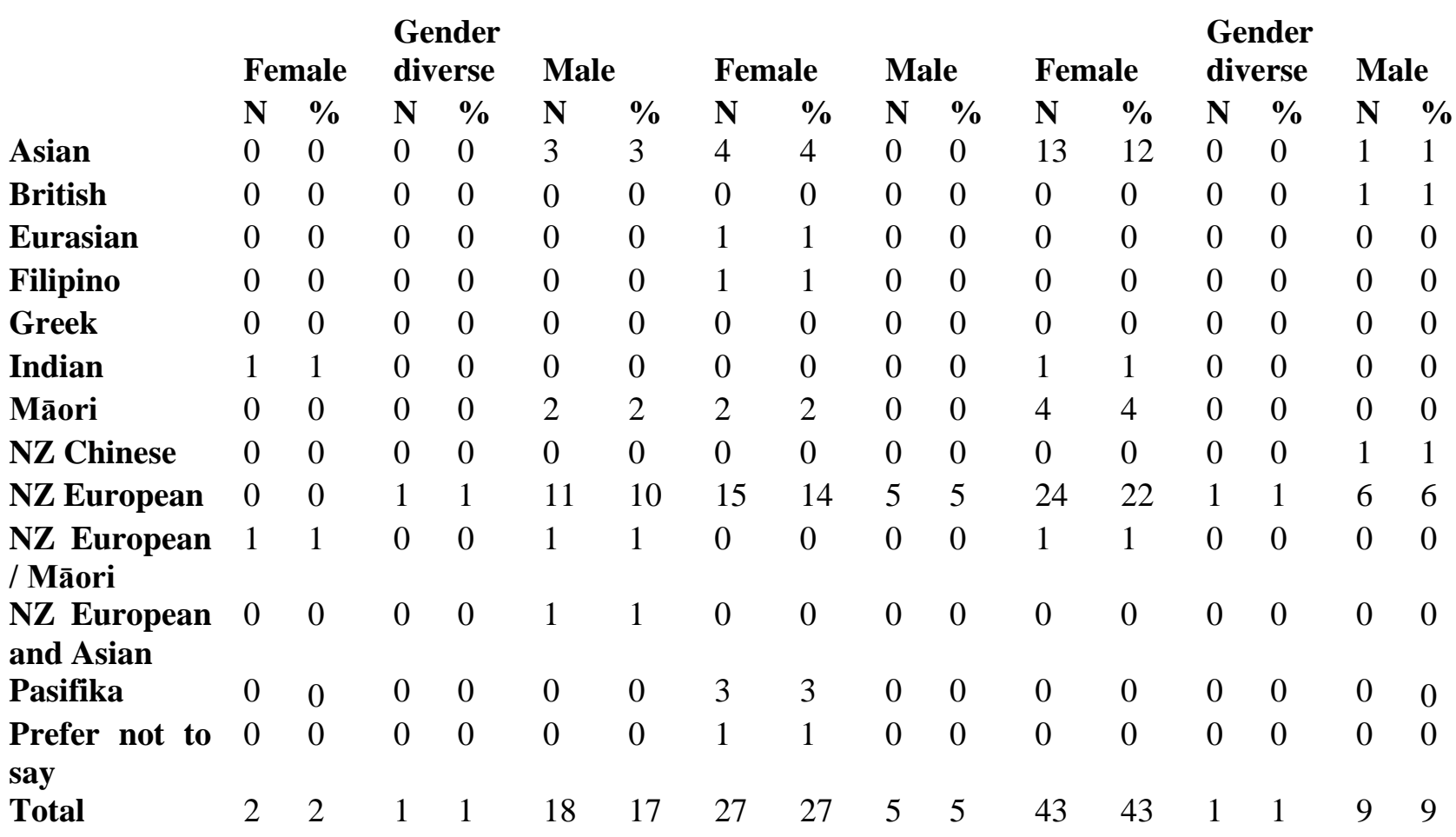


The participants reported working in a range of sectors and 28 participants reported working in more than one sector. The 10 most common sectors were retail trade (26 per cent), healthcare and social care (18 per cent), education and training (15 per cent), accommodation and food services (11 per cent), administrative and support services ( 9 per cent), public administration ( 7 per cent), construction (4 per cent), arts and recreation ( 5 per cent), hospitality ( 4 per cent) and wholesale trade (4 per cent).

Forty-four per cent of study participants were in full-time tertiary education, 13 per cent were in parttime tertiary education, 20 per cent were in secondary school education and 23 per cent were working full-time.

\section{Training}

Participants were asked about any health and safety training they had received when starting work; and 53 per cent reported receiving less than one day's training, with 34 per cent reporting that they had received training on the first day of work. However, 32.7 per cent of respondents reporting not receiving any health and safety training.

Respondents were also asked how confident the health and safety training they had received made them feel to do their job safely. Just under half ( 49 per cent) reported that the training made them feel confident; 26 per cent gave a neutral response and 29 per cent of respondents felt 'not confident'. Responses to an open question about the training they received were varied, including: "having a colleague with you to sign off was helpful"; assumption of prior training was made "and there were still things I was not trained in" and a lack of seriousness towards training where "it was run through quickly and wasn't made to seem very important".

While respondents were asked about training and how confident this made them feel, there were no significant statistical differences found regarding the amount of OHS training and confidence. Comments made in relation to an open-ended question with regard to how confident the training made the respondents feel about working safely. This question was thematically analysed and factors, such as working in a low-risk environment, personal knowledge levels, having resources available were identified as being an employee's responsibility. Negative factors included insufficient training, highrisk environments and safety training not being seen as important.

\section{Health and Safety Valued by Employer}

Participants were asked if they felt health and safety was valued by their employer and while 57 per cent said yes, 35 per cent said somewhat and eight per cent answered no. When asked which factors gave this impression of value, the results in Figure 1 are presented. For those that said 'other', comments made included "having a she'll be right attitude", "no evidence of health and safety representatives", "no reporting of a violent patient incident" and "none". 


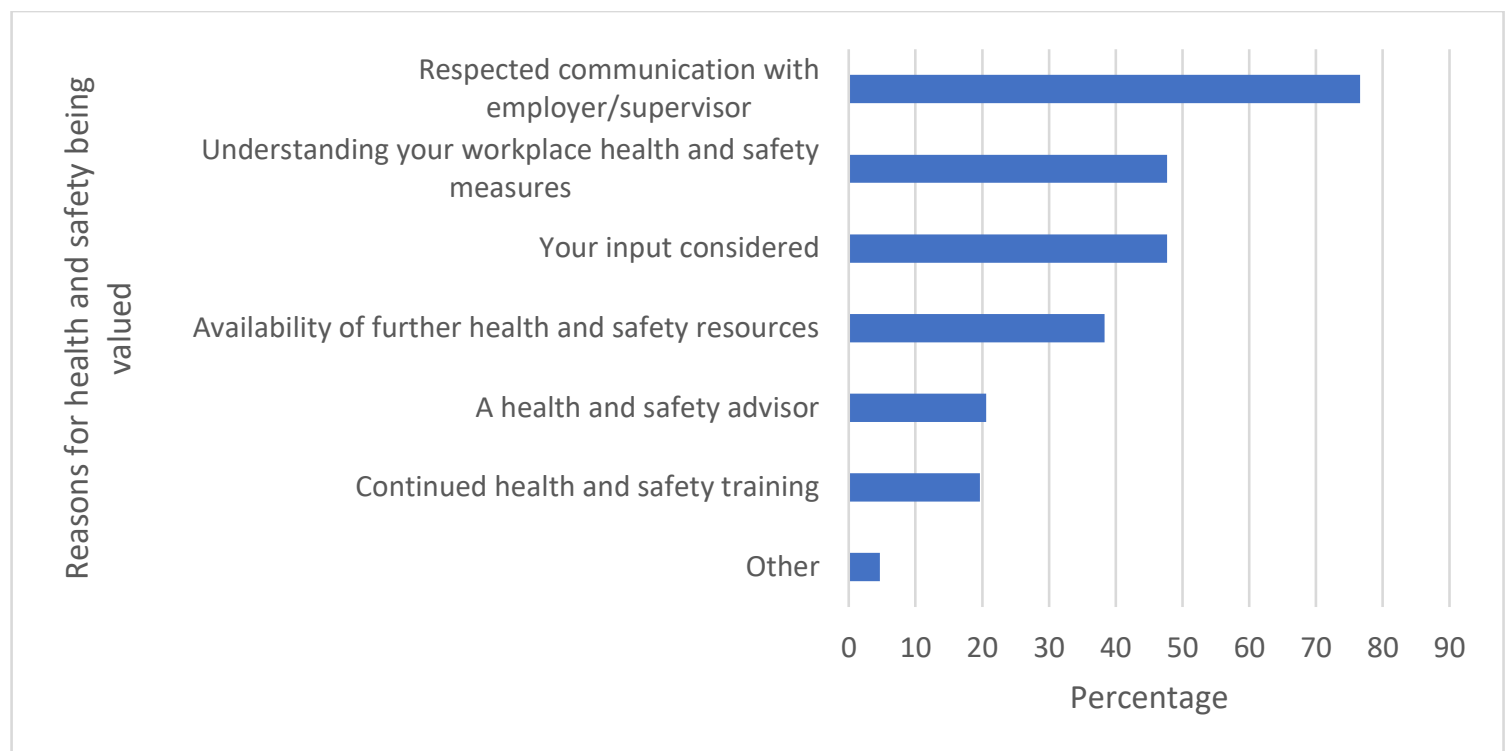

Figure 1: Why participants felt health and safety was valued by their employer

\section{Confidence}

Participants in the survey were asked how confident they would feel about speaking up about a health and safety issue on a Likert scale ranging from 1-5. The majority of respondents (63 per cent) were classified as confident as they ticked 1 "very confident" and 2. A further 20 per cent were described as neutral and 17 per cent were described as not being confident. When asked who they would feel confident to talk to about health and safety issues, 81 per cent said their boss/manager, 81 per cent their co-workers, 62 per cent friends and family, 59 per cent their supervisor, 35 per cent their head of department and 28 per cent their health and safety advisor; only one participant reported no one. Participants were then asked what factors would encourage them to speak up about workplace health and safety issues, the results are presented in Figure 2.

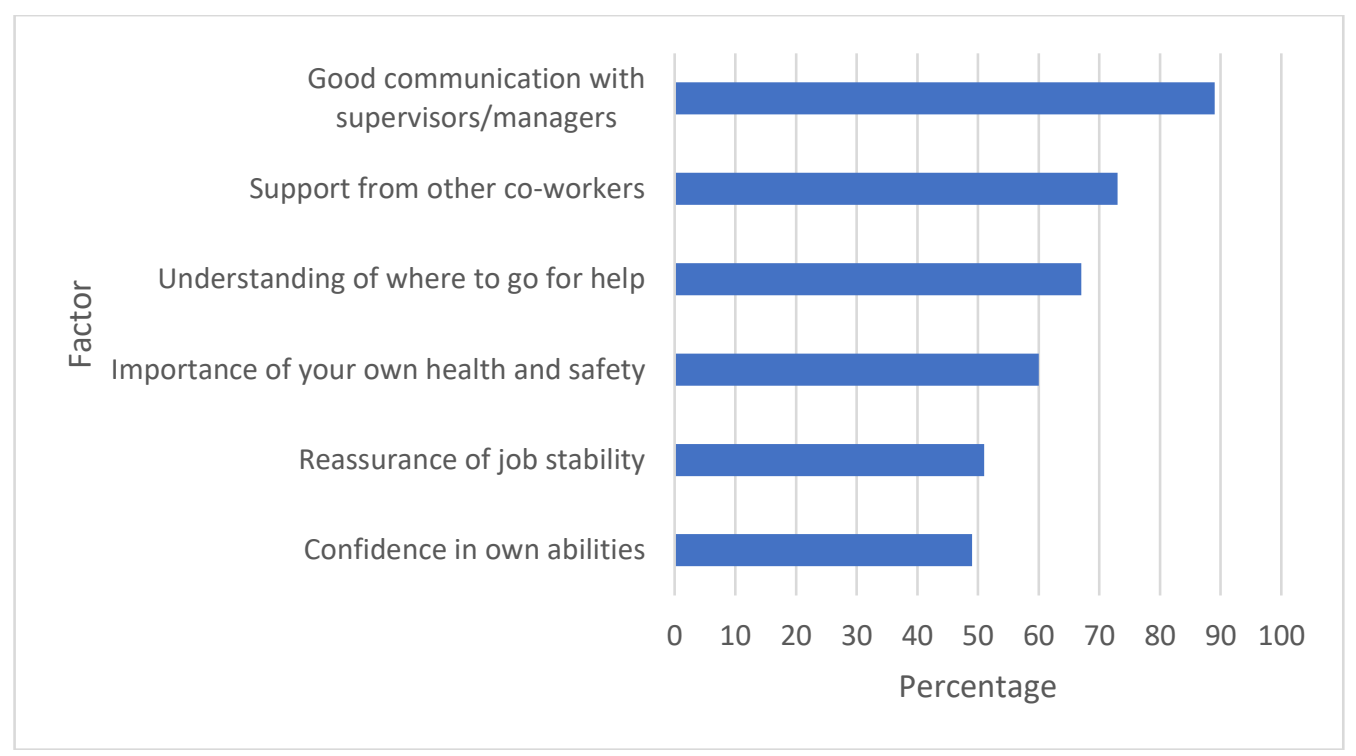

Figure 2: Factors that would encourage you to speak up about health and safety 
Additionally, when asked what would deter participants from speaking up about a health and safety issue, the data is reported in Figure 3. For those that reported 'other', comments included "knowing it won't be followed up" and "negative response, would actively ignore my advice". These data were further analysed to identify if there were any significant associations between confidence and gender, ethnicity and age. The analysis identified that the numbers reporting a lack of confidence significantly decreased as age increased $(\mathrm{p}=0.03)$.

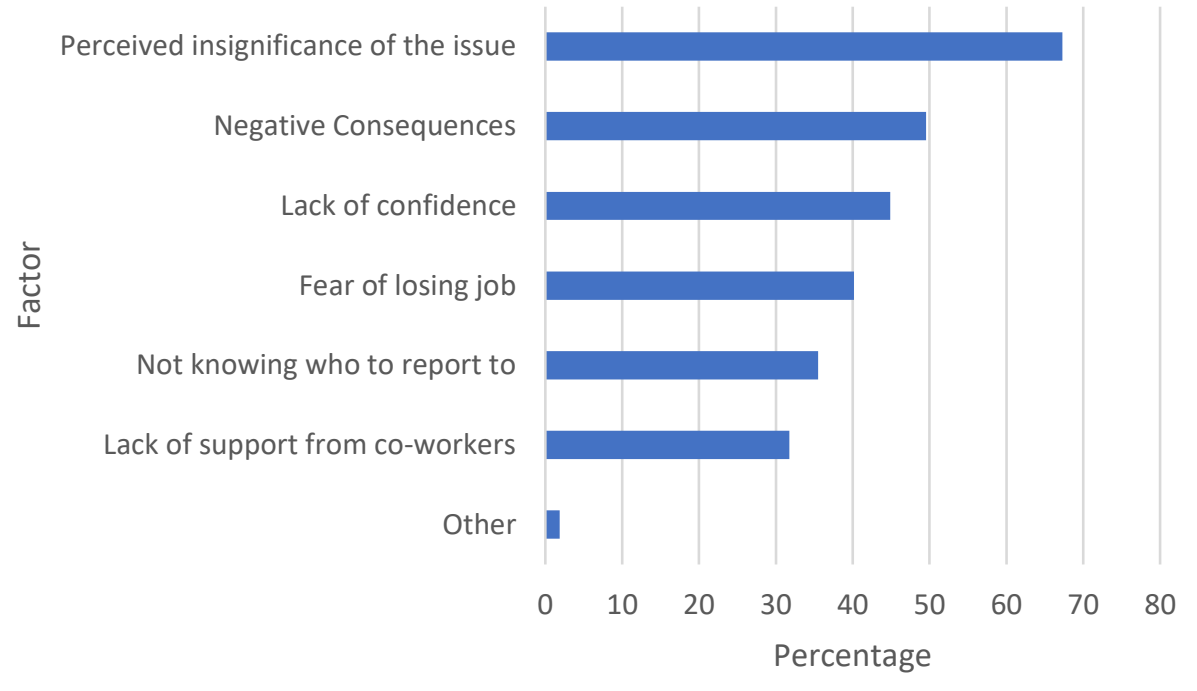

Figure 3: What factors would deter you from speaking up about a workplace health and safety issue

Stress

Participants were asked to rate how often they felt stressed or overwhelmed at work using a 5-point scale of $1=$ never and $5=$ always. The average score was 3.54 from participants. When asked about sources of stress at work, the results are presented in Figure 9. For those that replied 'other' (eight per cent), comments included "depression and anxiety", "lack of confidence in own performance and ability", "fear of failure", "bullying from supervisor", "being busy every day", "unsure if I am doing things right or making mistakes", "bad management" and "too few hours".

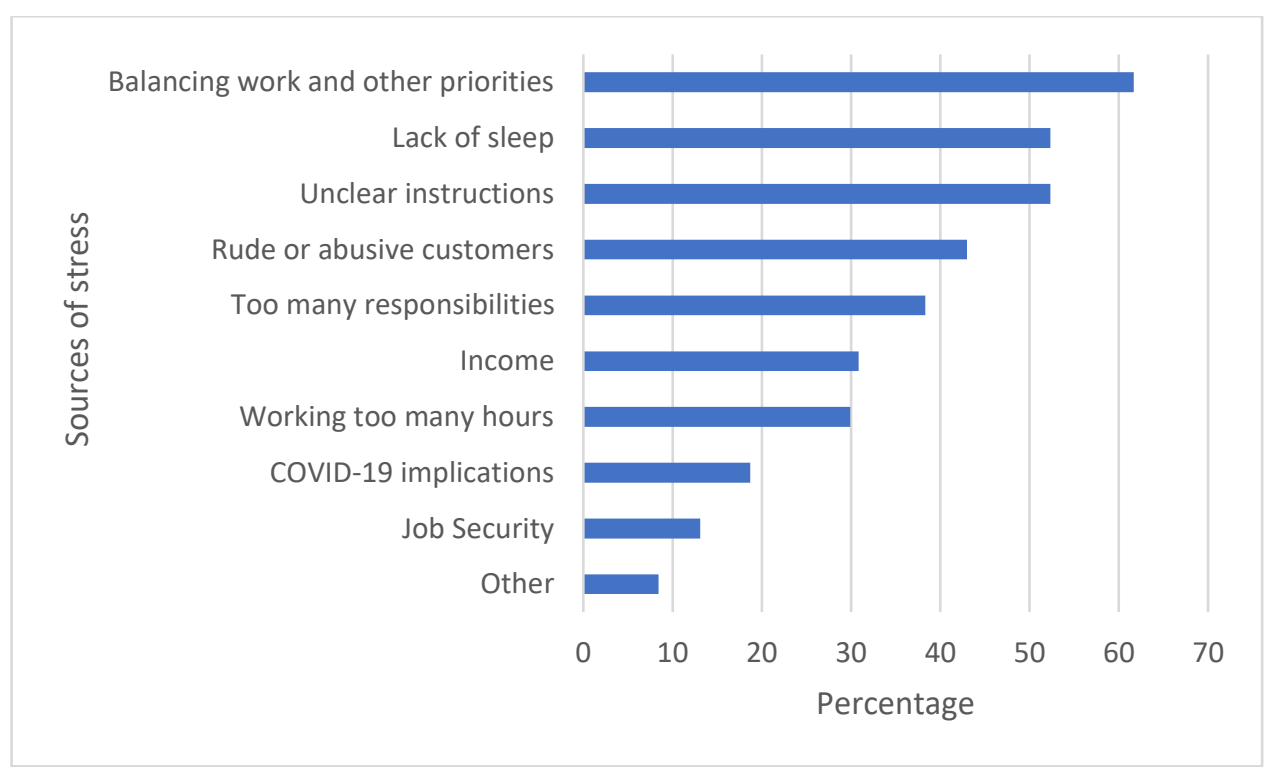




\section{Figure 4: Sources of stress at work}

Further analysis examined how often stress impacts on ability to follow health and safety protocols Within the sample 31 per cent said 'never', a score of 1, 31 per cent scored 2, 26 per cent scored 3, 15 per cent scored 4 and three per cent scored 5 - always. Further analysis using Chi-squared found that there was an association between feeling stressed and overwhelmed at work and ability to follow health and safety protocols $(\mathrm{p}=0.05)$.

\section{Suggestions for making younger people safer at work}

The final question of the survey was an open-ended question asking for suggestions that might make younger workers feel safer. In total, 31 (29 per cent) of participants responded to this question and the results were thematically analysed. From these, four themes appeared.

- Clear and open communication

The need for a clear and open dialogue between workers and employers, including clear instructions, but also the opportunity to share ideas and suggestions in a more participatory way. This would help to give workers the confidence to bring up questions when they do not understand or query tasks that they think are unsafe.

- Improved health and safety resources

Access to health and safety advisors and protocols as well as better team engagement on the topic of health and safety. It was also thought that mentorship, either through health and safety representatives or other more experienced staff, would be helpful.

- Assumption of knowledge

There appears to be an assumption that young workers start work with existing health and safety knowledge, but this is often not the case. Moreover, when changing jobs, it is also assumed that knowledge is transferred to the next job. What this might suggest is that our young people need educating in workers' rights and health and safety before entering the workplace

- Continuous and in-depth training

The fourth and final theme was around ensuring that there is continuous and updated training for individuals at work.

\section{Discussion and Conclusions}

\section{Demographics}

As an exploratory study, this was a small sample of 108 participants, of which females made up the majority, two-thirds versus one-third male. In relation to age, the majority of respondents were 21-24 years. In relation to ethnicity, the majority were New Zealand European followed by Asian. While exploratory, it was not aimed to represent the diversity in the New Zealand population, but will inform future study design. As Hanvold et al. (2018) identified, young workers are not a homogenous group 
and, from this study, there is a clear need to look at different work types, including full-time, part-time, full-time study with part-time work, part-time work with part-time study and other combinations.

The participants were also predominantly female so, therefore, a future need for a more balanced sampling strategy. However, the statistical analysis did not identify any gender differences in the responses. The same can be said for ethnicity from this sample but future research needs to stratify to ensure all ethnic groups in New Zealand are represented.

\section{Training}

While 50 per cent of the sample reported less than one day's training in health and safety, 32.7 per cent reported receiving no training in health and safety, even though this a requirement for all PCBUs (person conducting a business or undertaking) to provide "any information, training, instruction, or supervision that is necessary to protect all persons from risks to their health and safety arising from work carried out as part of the conduct of the business or undertaking" (Health and Safety at Work Act 2015). Employers and superiors in workplaces have a significant influence on the views of young workers towards health and safety (Safe Work Australia, 2020). Therefore, enhancing the knowledge and prioritisation of effective health and safety training to all levels of staff is incredibly important.

Training plays a huge role in young workers' and any workers' ability to safely work in an organisation. In our survey, participants responded to multiple questions surrounding training. We identified recurring themes of feelings of safety in the workplace by asking participants to expand on the question: "How confident did this training make you feel in your ability to do your job safely?" While responses differed, there were recurring concerns about what these young workers were feeling at work. The most common responses were that employers assumed 'employee responsibility' and 'insufficient training'. If assumptions are made on the knowledge levels of health and safety, this could put young workers in a vulnerable position.

Young workers may often be assumed to know about health and safety protocols because of previous employment or perceived competency. Making assumptions about the skill levels of young employees may lead to an increased exposure to risk. Providing effective health and safety training and information can help both employee and employer develop positive notions around health and safety, making healthy and safe working normal (Health and Safety Executive, 2012). Shifting employers' attitudes towards young workers' potential capabilities is likely to develop the communication skills between young workers and their employers. While young workers are responsible for ensuring their health and safety does not' negatively impact on the safety of others, young workers are at a higher risk of workplace-related harm due to a possible lack of experience and awareness, (Safe Work Australia, 2020). The responsibility and knowledge of health and safety protocols cannot be assumed in young workers. This assumption of perceived competency was a key theme in the majority of the survey questions surrounding training.

Improving health and safety training in the workplace is not a linear process. For instance, in our survey, we posed the question of how confident participants were to speak up about a health and safety issue/hazard. Within the answers to these questions, there were significant concerns on unresponsive management, and the importance of a good team environment. Communicating concerns and understandings of health and safety in a workplace is incredibly important. A study on the effectiveness of occupational health and safety training found that improving communication between management and workers, especially when relating to the perception of convenience, was significantly important (Ricci et al., 2016). If there is a lack of clear and open communication between management and workers, it may be difficult to convey any health and safety concerns from either side. From our survey 
results, health and safety training protocols, in most, if not all industries, are in need of desperate reorientation. Bridging the gap between business management and worker communication may contribute to the increased value of health and safety training in the workplace. It is hoped that this will reduce the vulnerability of young workers.

Organisations and workplaces need to provide appropriate training and information for their employees to ensure they have the ability to work safely (Employment New Zealand, 2021). Otherwise, continuing to hire young workers with possibly limited or no experience in a workplace is only going to make young workers more vulnerable. While there may be a perception that it is a waste of time and effort to provide training for young workers as they may not be working long-term, employers must remember that there is a requirement for all employees to receive training to enable a safe and healthy work environment. Furthermore, employers need to take into consideration how stress impacts young people, especially those who are also studying.

\section{Stress}

While traditionally health and safety and occupational stress have been separated when examining workplace risks, in the context of young workers, there is a need to look at the influences of work and study on health. Stress affects how we work safely in many ways, especially when considering the impacts stress has on the body, both mentally and physically. How stress is managed in a workplace is vital for ensuring the safety of young workers. For instance, our survey found that 51.4 per cent of participants stated that a lack of sleep had contributed to feeling stressed at work, alongside 29.9 per cent reporting that working too many hours contributed to this also. Numerous studies have found the connection between lack of sleep and inability to function normally. This is likely to increase risk to our young workers including the 77 per cent of participants in the study who are both working and studying.

If employees were to, more regularly, consider the impacts of these two factors (lack of sleep and working too many hours) on stress, it is possible that workplace-related harm may reduce. For example, training students in stress management techniques helped them to manage stress more effectively inside and outside of education (Pascoe et al., 2020).

Therefore, it is not illogical that implementing the use of stress-management skills/techniques as a part of health and safety training could help reduce the vulnerability of young workers in the workplace. As stress is something that everyone deals with at some point in their lives, the role of management is incredibly important. Participants were asked to give suggestions on how to improve feelings of safety at work. The top three suggestions were clear and open communication, improved health and safety resources, and continued and in-depth training. These, alongside possible stress-management skills, could help young workers feel less stressed at work and, ultimately, at a lower risk for workplacerelated harm.

\section{Confidence}

According to international literature, young workers' perceptions of the importance of workplace health and safety is made out to be somewhat skewed, if we consider how often the notion of young workers' attitudes towards health and safety infer a degree of naivety. While to a certain degree, this may be the case, the attitudes of young workers vastly differ in terms of their age, industry and previous experience. It is somewhat unreasonable to assume the same level of competency across all young workers, especially as young workers, aged 15-24, make up a significant proportion of the workforce (Safe Work Australia, 2020; Hanvold et al., 2018). 
The impact of lower confidence levels, lack of guidance and communication between young workers and employers are a concern in relation to young workers' vulnerability. As discussed in the results section, in a survey question about job confidence after health and safety training, 29 per cent of participants felt a rating of 4-5 on the Likert scale of $1=$ confident to 5 not confident at all, with a large majority at 2-3. It is perceived from these results that workplaces need to rethink their current health and safety training measure in order for young workers to feel confident, and to be able to do their jobs safely. A major consideration of our study was the need to make health and safety training build confidence in New Zealand viable for all New Zealanders.

Alongside an individual's perceived confidence in their ability to do their job, the very act of speaking up about a health and safety issue can be almost seen as essential for a healthy and safe work environment. In our survey, only 45.8 per cent of participants stated that they felt very confident in their ability to speak up about a health and safety issue/hazard. It would be helpful to identify ways of improving this confidence. A study by Okun et al. (2016) on young workers' risk found that young workers were more likely to change jobs and have multiple employers in the course of their working lives as compared to older workers. Such shifts mean that young workers are more likely to be faced with new hazards/risks. Therefore, it is important to integrate relevant and ongoing health and safety knowledge for young workers. Additionally, Clarkson et al. (2017) identified that many young workers report health and safety issues to their family who, on the whole, have little impact on the workplace.

Continuation of health and safety training throughout a young workers' time at a workplace was a recognised theme in many of the questions throughout our survey, in relation to training, stress and confidence. A study looking into healthcare professionals' ability to speak up for patient safety found that hesitancy to speak up about an issue was not only a contributing factor towards communication errors, but the perceptions of a risk was a main prerequisite for speaking up (Okuyama et al., 2014). These findings and feelings of hesitancy from this study of healthcare professionals can be applied to our research on vulnerability in the workplace for young workers as young workers tend to have less experience in workplace environments, compared to older workers. This lesser experience may be perceived as a negative thing where, if inherently young workers are more vulnerable if their health and safety is not considered.

Young workers often perceive themselves to be confident in their own abilities, yet are reluctant to bring up issues with their managers (Worksafe, 2018). Communicating this and building confidence to speak in future health and safety training is only a step towards reducing potential risk for young workers for workplace-related harm.

Changing the dynamics of health and safety in the workplace is a multidimensional consideration that will take considerable effort at all levels of a system. What can be inferred from the results of this study is that there is a serious need for how health and safety is treated in workplaces in New Zealand. Without a change to the perceptions of health and safety training, young workers will continue to be vulnerable.

\section{Limitations and future research}

We chose the age group of 16-24 despite the fact there is considerable research showing young people starting work earlier. We decided on this age group due to ethical considerations surrounding children (i.e. 15 and under), therefore, our results may not truly represent all working adolescents' perspectives of workplace health and safety, especially those working more informally, such as babysitting. 
Due to the small sample size, another limitation was not being able to stratify the sample based on gender, age and ethnicity, but it has given an insight into the different work categories to which young people belong, including full-time, part-time, education and part-time and informal work, such as babysitting. The short window of time in which this research project was conducted may lead to a degree of information bias, and a higher likelihood of mainly university and affiliated participants. Potentially, with a longer period of time, further groups/types of young workers may have been reached.

For future research, to better understand the voice of young workers, there needs to be more focused qualitative research - a mixed-methods approach, including survey work across different sectors. It may also be helpful to target certain industries and ensure different types of young workers within different sectors are represented, including those under 16 years of age.

This was an exploratory study to learn more about young workers' experiences in the workplace, especially in the context of health and safety, as well as create knowledge which can be useful for future research. The study has identified the importance of improving the health and safety for young workers in all industries. Training needs to be seen as an investment by employers even if the workforce is transient. However, open communication is key to building confidence in young workers and strengthening the way in which health and safety is enacted in the workplace.

\section{References}

Auckland Co-Design Lab. (2016). The Attitude Gap Challenge: a South Auckland Employment and Skills Challenge. https://static1.squarespace.com/static/55ac5ee5e4b08d4c25220f4b/t/5ab37c25758d46f90e271 $\underline{\mathrm{b} 4 \mathrm{~d} / 1521712213914 / \text { Attitude+Gap+Challenge+Final+report.pdf }}$

Bohle, P., Buchanan, J., Cooke, T., Considine, G., Jakubasuskas, M., Quinlan, M., Rafferty, M., \& Ryan, R. (2008). The evolving work environment in New Zealand: Implications for occupational health and safety (Technical report 10). National Occupational Health and Safety Advisory Committee

(NOHSAC). https://www.sit.ac.nz/Portals/0/upload/Evolving\%20work\%20environment\%20in\%20New\% 20Zealand\%20implications $\% 20$ for $\% 20$ occupational $\% 20$ health $\% 20$ and $\% 20$ safety; $\% 20 \mathrm{NOHS}$ AC\%20technical\%20report\%2010.pdf

Clarkson, L., Blewett, V., Rainbird, S., Paterson, J. L., \& Etherton, H. (2018). Young, vulnerable and uncertain: Young worker's perceptions of work health and safety. Work, 61(1), 113-23.

Department of Labour. (2007). Youth in the labour market.

Employment New Zealand. (2021). Health and safety at work. https://www.employment.govt.nz/workplace-policies/health-and-safety-at-work/\#scrolltoworkplace-health-and-safety-programmes

Hanvold, T. N., Kines, P., Nykänen, M., Thomée, S., Holte, K. A., Vuori, J., Wærsted, M., \& Veiersted, K. B. (2019). Occupational safety and health among young workers in the nordic countries: a systematic literature review. Safety and health at work, 10(1), 3-20. 
Health and Safety Executive. (2012). Why is health and safety training important? https://www.hse.gov.uk/treework/training-is-important.htm

MBIE (Ministry of Business, Innovation and Employment). (2018). Health and safety at work strategy 2018-2028. https://www.mbie.govt.nz/assets/69361d5a98/health-safety-at-work-strategy2018-2028.pdf

Okun, A. H., Guerin, R. J., \& Schulte, P. A. (2016), Foundational workplace safety and health competencies for the emerging workforce. Journal of safety research, 59, 43-51.

Okuyama, A., Wagner, C., \& Bijnen, B. (2014), Speaking up for patient safety by hospital-based health care professionals: a literature review. BMC health services research, 14(1), 1-8.

Pascoe, M. C., Hetrick, S. E., \& Parker, A. G. (2020). The impact of stress on students in secondary school and higher education. International Journal of Adolescence and Youth, 25(1), 104-112.

Ricci, F., Chiesi, A., Bisio, C., Panari, C., \& Pelosi, A. (2016). Effectiveness of occupational health and safety training. Journal of Workplace Learning. Journal of Workplace Learning, 28(6), 355-377.

Safe work Australia. (2020). Young workers. https://www.safeworkaustralia.gov.au/playlists/youngworkers

Statistics New Zealand. (2020b). Injury statistics - work-related claims: 2019. https://www.stats.govt.nz/information-releases/injury-statistics-work-related-claims-2019

Statistics New Zealand. (2020a). Youth labour dynamics. http://archive.stats.govt.nz/browse_for_stats/income-andwork/employment_and_unemployment/youth-labour-market-dynamics-NZ.aspx

WorkSafe. (2018). Young People in the workplace. https://www.worksafe.govt.nz/managing-healthand-safety/businesses/young-people-at-work/ 